\title{
Coronavirus: A study on possible treatment
}

\section{Amitabha Chanda*}

\author{
* Faculty (Retd.), Indian Statistical Institute. \\ Communication address: \\ Dr. Amitabha Chanda, \\ P36/1, Manasbag, Kolkata 700056, India \\ Email: amitabha39@gmail.com \\ Phone: (0091)(33) 25537298 (Resi.), (0091) 9830855481 (Mobile \& Whatsapp)
}




\begin{abstract}
In the present paper some basic study so far done by scientific community regarding SARS-COVID-19 is presented. Along with these opinions, expressed by different persons and their significance are examined in detail. Possibility of a few drug to combat the attack of SARS-COVID-19 is studied.
\end{abstract}




\section{Introduction}

The first $O C 43$ strain of the human $\beta$ - coronavirus family was characterized in 1967 as agents of human respiratory diseases. Until 2002, the new disease identified, Severe Acute Respiratory Syndrome (SARS), is caused by a human $\mathrm{CoV}$. It surfaced in Southern China and Hong Kong. Though $S A R S-C o V$ is known for more than a decade,but its present manifestation has created a lot of concern over the world.

\section{Present Global Situation}

The first report of $S A R S-C o V$ came from China at the beginning of 2020 . Initially, the rate of propagation was slow. Gradually, it gained momentum and number of afflicted person and death grew exponentially.

From China it spread over more than 190 countries one by one. In the third week of March 2020 China has been able to contain the onslaught, but other nations are in bewildered situation. The most affected country is Italy. In a nation of about 60 million population 4000 deaths has been reported as against 3400 from China.

Highly advanced country like USA has not yet been able to restrict its onslaught. Everyday large number of death is reported in USA also.

\section{$3 \quad$ Its Impact and Related Idea}

WHO has termed this onslaught as Pandemic. It has taken toll of economy, health and all other sectors of human society. National leaders are unable to decide if closure of schools, colleges, factories, entertainment centers is needed or not. Closure would create number of problem and so also running the shows.

However the objective of the present paper is to discuss possible ways to protect man from this attack. For this purpose I would like to tabulate the following information.

1. Japan has recently invented a new drug for flu in the brand name of Favipiravir, also known as $T-705$, Avigan and Favilavir. Chinese doctors have reported that Favipiravir, in combination with Tocilizumab, is effective against $C O V I D-19$.

2 . Before the introduction of Favipiravir similar drugs like Paritaprevir, Simaprevir, Grazoprevir were in the market. Those are for treatment of Hepatitis-C [1].

3 . Drugs for treatment of Hepatitis-C contain $S$ in their chemical structure. These are basically sulphonamide with small variation in molecular formula. 
4. Unlike Paritaprevir, Simaprevir, Grazoprevir Favipiravir has Fluorine in its structure in place of Sulphur.

5 . Fluorosis is extensively reported in few areas in West Bengal of India, like districts of Purulia, Bankura, Birbhum and the Basirhat area in the discrete of N. 24 parganas. Extensive study was conducted on this subject by Dipankar Chakraborty et. al [2]. It was found that the subsoil aqua layer of these areas contains considerable amount of Fluorine. Recently no outbreak of COVID - 19 has been reported in these areas.

6 Number of infected person in West Bengal is not very large. In fact small number of infected persons in West Bengal come from Fluorine rich areas. It may be due to presence of fluorine in subsoil water.

7 The small and highly reactive atom of Fluorine has evoked a large scale interest among researchers. The enhanced membrane permeation and increased binding affinity of fluorinated drug to target protein [3] may increase its clevaging capacity of S-protein of COVID-19.

\section{Some Ideas}

In view of the above logic the author feels the need for examining if Fluorine in some form is able to restrain the onslaught of this virus. Medical scientists may suggest some form, but the author would like to make an humble suggestion.

Atorvastatin is a widely used drug for hypertension and diabetes. It has Fluorine in its structure. Rampant use of this drug may not be advisable. But the author feels that doses of Atorvastatin as prophylactic and as a drug against Corona may be studied. Its use as a drug in combination with Favipiravir for treatment of a Corona patient may also be studied. Aldrich [3] produces number of drugs like iodo fluoro methylquinoline, amino fluoro methylquinoline. Experts may find a suitable one from those drugs and advise its in place of Atorvastatine to have greater effect.[4]

Some other statins like fluvastatin has F in its structure. At this stage I would like to discuss another item. HRP (Hroseradish Peroxidase) has the capacity to catalyse reduction of $\mathrm{H}_{2} \mathrm{O}_{2}$ in body and clevage S-protein of COVID-19. HRP has a heme structure. I am not sure but if possible a Fluorinated HRP [5] may be a better solution.

\section{References}

1 Steven H. Liang, Adam G. Southon, Benjamin H. Fraser Anwen M. KrauseHeuer Bo Zhang Timothy M. Shoup, Rebecca Lewis, Irene Volitakis, Yifeng Han, Ivan Greguric, Ashley I. Bush. Novel Fluorinated 8-Hydroxyquinoline Based Metal Ionophores for Exploring the Metal Hypothesis of Alzheimers Disease. ACS Med Chem Lett. 2015 Sep 10; 6(9): 10251029. 
2 Piyal Bhattacharyya and Aloke C, Samal. Fluoride contamination in ground water, soil and food stuffs India and associated health risks: A review.Research Journal of Recent Sciences, vol. 7(4), pp 36-47, April 2018.

3 Poonam Shah \& Andrew w. Westwell. . The role of fluorine in medicinal chemistry. Journal of Enzyme Inhibition and Medicinal Chemistry, Octobor 2007; 2295. 2015. .pp 527-540/

4 https://www.sigmaaldrich.com/chemistry/chemistry-products.html

5 https://patents.google.com/patent/WO2009140215A2/en

6 Guido R. Lopes, Diana C. G. A. Pinto and Artur M. S. Silva. Horseradish peroxidase (HRP) as a tool in green chemistry. RSC Adv.,37244. 2014. 